\title{
Mobility Management Strategy Based on User Mobility Patterns in Wireless Networks
}

\author{
Wenchao Ma, Member, IEEE, Yuguang Fang, and Phone Lin, Senior Member, IEEE
}

\begin{abstract}
Mobility management plays an important role in wireless communication networks in effectively delivering services to users on the move. Many schemes have been proposed and investigated extensively in the past to improve performance. Profile-based scheme (PBS) is one of them, which uses the user routine information stored in the user profile to reduce location update signaling traffic. To improve PBS performance, in this paper, a new location management scheme called the mobilitypattern-based scheme (MPBS) is proposed, which incorporates the time information in the user profile so that a user's current location can be determined by his/her movement state and the current system time. Extensive simulations have been carried out, and the results show that the MPBS scheme can mitigate the signaling traffic load significantly while reducing paging delay at the same time.
\end{abstract}

Index Terms-Location management, mobility management, personal communication systems, user mobility pattern (UMP).

\section{INTRODUCTION}

$\mathbf{T}$ HE VISION of anytime and anywhere communications is the target at which future-generation wireless communication systems aim. At the beginning of the 1970s, mobile users could only roam locally or regionally, while international roaming was possible only after the 1990s [1]. With the increase of mobile user population and mobility, and more demands on quality of service (QoS), the current mobility management scheme faces new challenges.

Currently, there are two protocols widely used in wireless systems. The IS-41 has been adopted in North America, and GSM MAP has been accepted in Europe and Asia [2], [3]. The basic mechanisms of these two schemes are very similar. Both of them manage the user mobility by a two-tier hierarchical database system consisting of home location register (HLR) and visitor location register (VLR) [5]. A mobile user permanently registers to an HLR with the user profile. The VLR stores user information currently residing in its charge area. When a user enters a new register area (RA) charged by a new VLR, the

Manuscript received December 27, 2004; revised August 12, 2005 and January 31,2006 . This work was supported in part by National Science Foundation Faculty Early Career Development Award ANI-0093241. The review of this paper was coordinated by Prof. T. Hou.

W. Ma is with the Lenovo Corporate Research and Development, Haidian District, Beijing 100085, China (e-mail: mawc@lenovo.com).

Y. Fang is with the Department of Electrical and Computer Engineering, University of Florida, Gainesville, FL 32611-6130 USA (e-mail: fang@ece.ufl.edu).

P. Lin is with the Department of Computer Science and Information Engineering, National Taiwan University, Taipei, Taiwan, R.O.C. (e-mail: plin@csie.ntu.edu.tw).

Color versions of one or more of the figures in this paper are available online at http://ieeexplore.iee.org.

Digital Object Identifier 10.1109/TVT.2006.883743 mobile terminal is required to send an update message to the new VLR. The new VLR will exchange information with user's HLR through a global-title-translation procedure and obtains the user's profile. At the mean time, the HLR also contacts the old VLR to deregister the user there. In the IS-41 or the GSM MAP protocols, the HLR can locate users in the RA granularity, so all the cells in that RA will be paged once a call request is received. Upon receiving the paging signal, the target mobile terminal will respond with an acknowledgment (ACK) message, and a connection can be set up following some procedures. Due to the information exchanges, the location management signaling traffic burden increases dramatically with the increase of the user population. Statistics data show that more than $90 \%$ signaling traffic is generated for location update in some highdensity urban areas [6]. In the $3 \mathrm{G}$ wireless communication system, high-speed multimedia services will be provided, and current RAs in the $2 \mathrm{G}$ systems may have to be partitioned further to meet the QoS requirements. It is obvious that the smaller RA size will exacerbate the network signaling burden further with the current mobility management schemes [4], [7].

From our daily life, we observe that many mobile users follow some fixed routines everyday. For example, a person drives to his/her office every morning along a road, stays in the office most of the day, and goes home after work along the same road; a mailman delivers mail along fixed routes everyday. If the network knows the mobile users' daily route information, then the location update signaling traffic burden can be mitigated. Based on this observation, a profile-based scheme (PBS) was proposed in [1] and [11]. In this scheme, a user's daily routine information is stored in the profile. If the user follows his or her itinerary well, then no location update is necessary, so that the update traffic is reduced. When a call arrives for that user, the RAs where the user could be in will be paged. In the Le-Zi update [12] scheme, the user movement history is sent to the network. The registered history data are used to construct a search tree for the user. We notice that a user mobility-patternbased mobility management scheme is also proposed in [13]. In the scheme, a user mobility pattern (UMP) is found or created based on the user's movement, and the probability that the user is in a cell is also derived based on the pattern. In those schemes, the paging can be implemented in all the RAs at the same time or operated one by one following a descending probability order. In the above schemes, the location update cost is reduced at the expense of increasing the total paging costs. A user who follows some daily routine may deviate from it because of road traffic, weather, or some other reason. In this case, the mobile terminal is required to report to the new VLR, just as with the IS-41 or the GSM MAP scheme. Therefore, the mobile terminal 
needs to store the list of RAs in the memory, which are updated or adjusted by the network periodically. In [11], the author did not consider the call-to-mobility ratio (CMR) impact on the PBS performance. In this paper, we show that the user CMR is very critical in the PBS, and the traffic savings can only be achieved under a certain limited CMR range. A new mobilitypattern-based scheme (MPBS) is also proposed in this paper. Compared to the study in [13], our scheme can adopt different paging strategies based on the user states so that the UMP cost can be minimized.

The new scheme we proposed is called user MPBS. In this scheme, the UMP is used to minimize the location update and paging traffic burden. The basic idea is to incorporate the time information in the mobility pattern profile; thus, different location update and paging schemes can be used based on the different user states. The simulation results show that the MPBS generates significantly less signaling traffic than both the IS-41 and the PBS schemes, and the paging delay in the MPBS is shorter too. In next-generation wireless communication systems, the service providers will provide the users with useroriented services so that the network resources are allocated according to the user service requirements. With the MPBS scheme, the advantage is that the system can predict the user's future location, and the resource can be assigned in advance for the users engaging in high-priority applications [8], [9].

The paper is organized as follows. In Section II, we introduce the virtual management network (VMN) concept and describe the architecture of the signaling network in a wireless communication network. Then, we give the detailed description of the PBS and MPBS in Section III. In Section IV, we estimate the signaling costs for all the three schemes and discuss the simulation network architecture and system configuration. The performances for all the schemes are demonstrated in Section V, and Section VI concludes the paper.

\section{System Description}

For next-generation wireless communication networks, different classes of users have different service requirements. Different priorities can be assigned to users based on their call history, mobility pattern, and willingness to pay for a higher QoS. In order to achieve this goal, the network needs to collect and store some extra user information in the user profile. For example, if a UMP is known, some resources or information can be prepared to the user in a certain area where the user will soon be. If the above additional workloads, such as information collection and dissemination, are incorporated into the current distributed wireless communication database system, the signaling and database retrieval traffic may overwhelm system process capacity. The delay resulting from the heavy traffic load may degrade system performance completely. In order to solve the problem, we propose the VMN concept.

Virtual Management Network (VMN) is an overlaying management network on top of the existing wireless mobile networks, which handles the intelligent features. In our scheme, a new function entity - mobility agent (MA) - is proposed. If we assume that the signaling network for a wireless mobile network is a common-channel-signaling network, such as the signaling system No.7 (SS7) [14], the MAs can be hardwarebased or software-based and are connected to both VLRs and HLRs; at the same time, the MAs are connected to each other. Each MA is in charge of a selected set of RAs and can be dynamically configured according to the network's up-to-date traffic burden. In the SS7, the MAs can be placed together or separately at the same level as local signaling transport point (LSTP) or regional STP (RSTP). If both LSTP and RSTP are equipped with MAs, we can say that the VMN is configured hierarchically. The VMN can also connect to an operations, administration, and management (OA\&M) center that maintains the networks and collects user information. In this paper, we concentrate only on the location management issue.

\section{PBS AND MPBS SCHEMES}

In this section, the PBS and MPBS schemes are introduced. More details about the PBS can be found in [11].

\section{A. Profile-Based Scheme (PBS)}

In PBS, the network maintains a record of each user's most likely itinerary list. It is assumed that the user's location distribution probability is known in advance. It can be provided by the mobile terminal or estimated by the network according to the user's calling history. The location list is stored in the switch, and the information required to update the list is stored within the mobile switch billing center.

The user's itinerary can be defined as follows. If $A_{i}$ is one of the RAs in the record list, the user's most likely itinerary can be defined as $\boldsymbol{A}_{k}$, where $k$ is the number of RAs in the set. The probability of a user being in an RA $A_{i}$ is $\alpha_{i}$. The system maintains a list of $\left(A_{i}, \alpha_{i}\right)$ pairs for some time interval $T$. The probability of a user being out of $\boldsymbol{A}_{k}$ is given as

$$
\kappa=1-\sum_{i=1}^{k} \alpha_{i} .
$$

If $\kappa>0$, there is probability of not finding the user in the list. If the user follows his/her daily itinerary strictly, namely, the user keeps roaming in $\boldsymbol{A}_{k}$, no registration is needed. When the user is out of the list, the mobile terminal is required to manually register to the network. Therefore, the terminal must keep a copy of the list. The list can be sent to the terminal by network and updated whenever some $A_{i}$ is added or deleted. When a call request arrives for the user, the RAs in the list are paged in the descending order of $\alpha_{i}$ until the user is found. Under this strategy, the database will know a user's exact location when it is outside $\boldsymbol{A}_{k}$. Indeed, when the user is out of the list, the PBS scheme works the same as the IS-41 or the GSM MAP. According to these procedures, the PBS can reduce the location update cost effectively at the expense of increasing paging cost. In [11], the authors studied the paging delay and the radio link cost under different lengths of the list. The paging delay was derived, given three known probability distributions, in terms of the number of the expected RAs needed to be paged before the user is found. In fact, it is intuitive that the total cost of the PBS scheme has a tight relationship with the user's CMR. With 


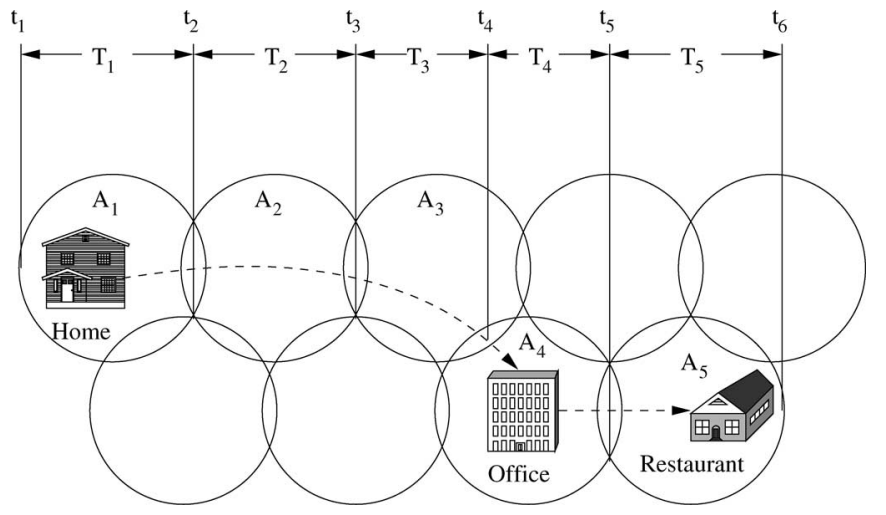

Fig. 1. Procedures in MPBS.

a small CMR, which means that the user has a relatively high moving rate over the call arrival rate, the PBS can reduce most update costs and perform well. However, for the users with high CMR, the paging cost will dominate, and the total costs of PBS may exceed those of the basic IS-41 or the GSM MAP schemes.

\section{B. Mobility-Pattern-Based Scheme (MPBS)}

The MPBS strategy can reduce the user update cost and attempts to limit the paging cost at the same time [15]. Comparing with the PBS, two more elements-the time a user entering $A_{i}\left(t_{i}\right)$ and the residence time in $A_{i}\left(T_{i}\right)$-are added in the user profile. The MA will maintain a list of 4-tuple $\left(A_{i}, t_{i}, T_{i}, \alpha_{i}\right)$ for each user. We assume that the cardinality of the set for a user is $k$. In the list, the tuples are not ordered according to $\alpha_{i}$ but to $t_{i}$. For example, if a user's profile includes all the location entries he/she may visit in $24 \mathrm{~h}$, the list is sorted by the time the user visits every $A_{i}$. Therefore, for $i \neq j, A_{i}$ and $A_{j}$ may be the same. In the MPBS scheme, we define $\varphi>0$ as the user out-of-pattern probability, which is given as

$$
\varphi=1-\sum_{i=1}^{k} \alpha_{i} .
$$

If $\varphi>0$, there is a probability that the user moves out of the RAs in the list. We say the user is out-of-pattern if this happens; otherwise, we say the user is in-the-pattern. Fig. 1 shows an example of the MPBS. If a user lives in RA $A_{1}$ and works in the office building which is in $A_{4}$, before reaching the office, the user will pass through $A_{2}$ and $A_{3}$. The user would also like to have lunch break in a restaurant which is in $A_{5}$. If the user follows the itinerary everyday and the information is stored in the profile, the network can locate the user based on the location profile and current time of a day.

To make the scheme clearer, we need to define the user behaviors more precisely. In the MPBS scheme, when users follow the UMP, the location update traffic can be reduced. There are two kinds of patterns for users to follow in the MPBS scheme. When a user enters $A_{i}$ at time $t_{i}$ and the residence time in $A_{i}$ is $T_{i}$, we say the user follows the time-sequence pattern. If the user enters and exits RAs following the $A_{i}$ order in the profile only, we say the user follows the sequence pattern. For example, an unexpected road congestion makes the user late for entering every RA to the office, but he still follows the same route as before. It is obvious that a user following the timesequence pattern follows the sequence pattern as well, while a user following the sequence pattern may not follow the timesequence pattern. Users may deviate from their daily routine for some reasons, so we need to find out how close a user is following the UMP. Mobile terminals for the next-generation systems should incorporate more intelligent functions. When a user roams in a network service area, we assume that the mobile terminal can record the RA ID and the RA entrance and exit times. We define the user's actual path information as the user actual path (UAP). The UAP can be used to update the UMP periodically. As the time elapses or the user crosses RA boundary, the mobile terminal can tell whether the user is following any pattern or not by comparing the UAP with UMP. The UAP is in the same format as UMP. If we do not consider the time information, the UAP can be expressed as $\boldsymbol{B}_{m}$, where $m$ is the length of the UAP. Without the consideration of time, we can compare the similarity of UAP with UMP by the edited distance [10]. We assume that the regular movement of a mobile user can be modeled as an edited UAP by allowing the following legal operations.

1) Inserting an RA $L$ at position $i$ of the UMP gives UAP: $A_{1}, A_{2}, \ldots, A_{i-1}, L, A_{i}, A_{i+1}, \ldots, A_{k}$.

2) Deleting the RA $A_{i}$ of the UMP gives UAP: $A_{1}, A_{2}, \ldots, A_{i-1}, A_{i+1}, \ldots, A_{k}$.

3) Changing an RA $A_{i}$ of the UMP to another RA $L$ of the UMP gives UAP: $A_{1}, A_{2}, \ldots, A_{i-1}, L, A_{i+1}, \ldots, A_{k}$.

As a result, the edited distance between a UMP and UAP becomes the sum of the editing operation weights. If the edited distance is less than a threshold, we can say that the user follows the sequence pattern, indicating the general moving intention of the user. For large systems with complex network topologies, the calculation of the spatial weights can be more involved. How to exactly assign and calculate the spatial weight is out of the scope of this paper. For simplicity and without loss of generality, we define the weight as follows:

1) the cost of inserting

$$
W_{I}= \begin{cases}1, & L \text { is the adjacent RA of } A_{i} \\ \infty, & \text { otherwise }\end{cases}
$$

2) the cost of deleting

$$
W_{D}= \begin{cases}0, & A_{1}, \ldots, A_{i-1} \text { have already been deleted } \\ 1, & \text { otherwise }\end{cases}
$$

3) the cost of changing

$$
W_{C}= \begin{cases}1, & L \text { is the adjacent RA of } A_{i} \\ \infty, & \text { otherwise. }\end{cases}
$$

Based on the above notations, we can define the user behaviors more precisely. When a user enters $A_{i}$, the user is said to follow the time-sequence pattern if and only if the following requirements are met: 1) $A_{i} \in \boldsymbol{A}$; 2) $\left|t_{i \text {,actual }}-t_{i}\right|<\Delta T$; and 3) $t-t_{i}-T_{i}<\Delta T$, where $t_{i, \text { actual }}$ is the actual time the user enters $A_{i}, t$ is the current system time, and $\Delta T$ is the time pattern threshold. The first 
TABLE I

USER STATES IN ONE RA

\begin{tabular}{|c|l|}
\hline State & Definition \\
\hline 1 & User follows the time-sequence pattern \\
\hline 2 & User follows the sequence pattern \\
\hline 3 & User does not follow any of the above two patterns but $A \in \boldsymbol{A}$ \\
\hline 4 & $A \notin \boldsymbol{A}$ \\
\hline
\end{tabular}

condition constrains the user in the profile, and the second and third conditions limit the user to enter and exit location area (LA) $A_{i}$ within some time threshold, respectively. If we assume that a UAP is $\boldsymbol{B}$, the edited distance between UAP and UMP is $d\left(A_{1}, A_{2}, \ldots, A_{N}, B_{1}, B_{2}, \ldots, B_{N}\right)$, and the edited distance threshold is $\Delta D$, where $N$ is the selected pattern length large enough to guarantee the comparison result accurate. We can say that the user follows the sequence pattern if: 1) $A_{i} \in A$; 2) $\left|t_{i \text {,actual }}-t_{i}\right|>\Delta T$ or $t-t_{i}-T_{i}>\Delta T$; and 3) $d\left(A_{1}, A_{2}, \ldots, A_{N}, B_{1}, B_{2}, \ldots, B_{N}\right) / N<\Delta D$. In the MPBS scheme, a user can be in one of the four states, as defined in Table I, when the user enters an RA.

The reason we define the four states is that the network can invoke different paging mechanisms for users in different states. A user needs to inform the network whenever the state changes. In the MPBS scheme, we assume that the user state information is included in the update message, so that the network will know the user's current state every time the mobile terminal sends an update message. When a user is in states 1,2 , or 3, no registration or state message needs to be sent as long as the user keeps the current state unchanged. If the user is in state 4 , the mobile terminal needs to update its location to the network every time the user enters a new RA. Then, in state 4 , the user needs to update the location every time he/she crosses an RA boundary. If we can collect the user daily routine information well enough that the user has a large probability in states 1,2 , or 3 , the update cost can be reduced. In the MPBS scheme, when a call arrives, the network can use different paging strategies based on the user's current state. If the user is in state 1, the network can determine which RA the user is in according to the current system time and page it. For example, the current time is $t$ and the user is in state 1 when a call request arrives, the network can retrieve the tuple matching $t_{i} \leq t \leq t_{i}+T_{i}$; then, the user can be located in $A_{i}$. It is possible that the user just moves out of the paged area and enters the next one. If the user does not change the state, only the next RA needs to be paged. If the user is in state 2 , the network knows which RAs the user is not in, according to the last location update. Since the user follows the sequence pattern, the network knows that the user must be in one of the RAs after the last updated one in the profile. Because there is no time information in state 2 , all the RAs the user could be in will be paged in the descending order of the probabilities $\alpha_{i}$. If the user is in state 3 , the network knows that the user is in $\boldsymbol{A}$, and all the RAs in $\boldsymbol{A}$ will be paged according to the descending order of $\alpha_{i}$ until the user is found, which is the same as in the PBS scheme. If the user is state 4, the network knows the user's exact location and pages the respective RA. In fact, when the user is in state 4, the MPBS scheme is exactly the same as the IS-41/GSM MAP scheme.

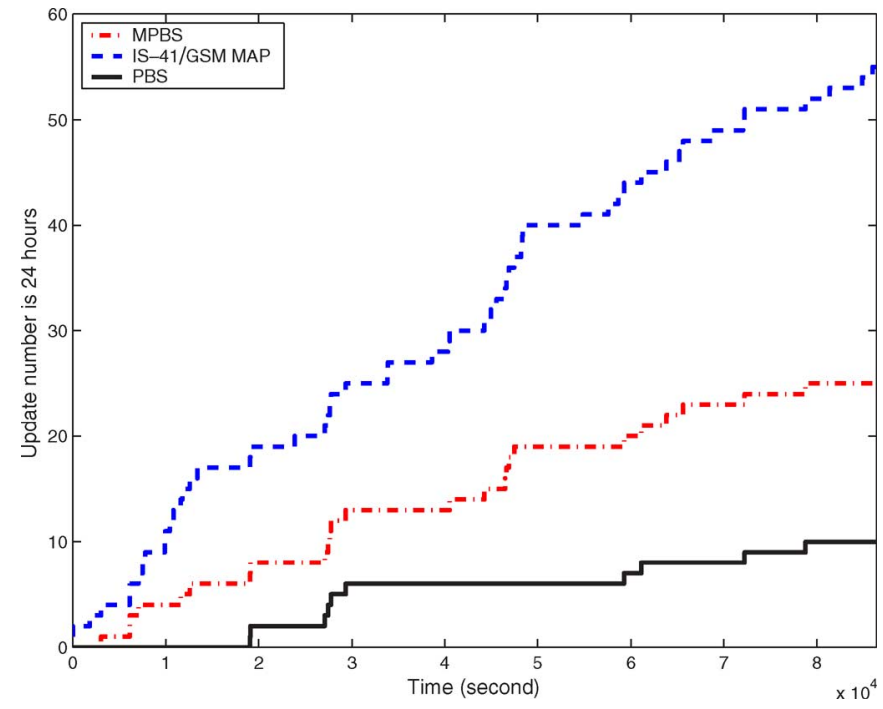

Fig. 2. Numbers of updates for three schemes in $24 \mathrm{~h}$ with a user residence time of $30 \mathrm{~min}$.

As we can see in Fig. 2, the IS-41/GSM MAP generates the most update messages, and the PBS scheme will generate the least traffic. The MPBS is in the middle of them. Although the MPBS results in more update messages than the PBS does, it reduces the paging cost dramatically and helps in achieving a total cost reduction, as we can see in the following.

\section{Performance Evaluation}

In this section, we carry out the performance analysis for our new scheme. To do this, we need to estimate the total signaling costs for the three location management schemes: IS-41/GSM MAP, PBS, and MPBS. Since the user behaviors and many other random factors affect the results significantly, it is hard to obtain the close forms, if any. Therefore, in this paper, we only present the general procedure to calculate the cost of each scheme and carry out the performance comparison through simulations.

We define $C_{u}$ as the cost for location registration and $C_{p}$ as the cost of paging one RA. In our analysis, we do not consider the cost for the call delivery processing because it is the same for all three schemes. We also want to point out that cost analysis is done between two consecutive calls as the call arrivals form a regenerative process [17]. For IS-41/GSM MAP scheme, the total cost between two consecutive calls are given in [16]

$$
C_{\mathrm{GSM}}=\frac{C_{u}}{\rho}+C_{p}
$$

where $\rho$ is the CMR. In the following analysis, we assume that there are $k$ RAs in the user's profile. For the PBS scheme, we define $E(k)$ as the average number of RAs that has to be paged before the user is found. Therefore, it is straightforward to know that the total cost for the PBS scheme is

$$
\begin{aligned}
C_{\mathrm{PBS}} & =\omega_{1} \frac{C_{u}}{\rho}+(1-\kappa) E(K) C_{p}+\kappa C_{p} \\
& =\omega_{1} \frac{C_{u}}{\rho}+[\kappa(1-E(k))+E(k)] C_{p}
\end{aligned}
$$


where $\omega_{1}$ is the probability that the user moves in or out of the profile between two consecutive call arrivals. The analysis of total cost for MPBS is more complicated. If we define $C_{p_{i}}$ as the paging cost for users in state $i$ and $\pi_{i}$ as the probability that the user is in state $i$ when a call arrives, the total cost of MPBS can be expressed as

$$
C_{\mathrm{MPBS}}=\omega_{2} \frac{C_{u}}{\rho}+\sum_{i}^{4} \pi_{i} C_{p_{i}}
$$

where $\omega_{2}$ is the probability of the user's movement that is needed to update the HLR. It is obvious that $\sum_{i}^{4} \pi_{i}=1$.

We need to derive $C_{p_{i}}$ to calculate the total cost for the MPBS scheme. In the MPBS, when the user is in state 1 , if a call arrives, the RA where the user is currently in will be paged according to the profile. However, it is possible that the user just moves into the next RA when the current RA is being paged. In order to make sure that the user is found, the next RA needs to be paged if no response is received in the predefined time in the current area. We assume that $t_{i \text {,actual }}-t_{i}$ follows the Gaussian distribution with zero mean and variance $\sigma$. Then, the probability that the user just moves to the next RA when a paging message arrives is $0.5-Q(\Delta T / \sigma)$. Since the paging expiration time is very short, we ignore the probability that the user crosses more than one RA during the paging. Based on the above analysis, we can obtain

$$
\begin{aligned}
C_{p_{1}} & =C_{p}+\left[0.5-Q\left(\frac{\Delta T}{\sigma}\right)\right] C_{p} \\
& =\left[1.5-Q\left(\frac{\Delta T}{\sigma}\right)\right] C_{p} .
\end{aligned}
$$

If a user is in state 2 when a call arrives, only the subsequent RAs need to be paged because it is known that the user is not in the first $m(m<k)$ LAs. Therefore, the probability that the user is in the subsequent LAs is conditional. If we assume that there are $m$ RAs being crossed before a call arrives and let $X_{m}=\alpha_{1}+\alpha_{2}+\cdots+\alpha_{m}$ and $k^{\prime}=k-m$, we can obtain the conditional probability distribution for the next $k^{\prime}$ LAs as $\alpha_{1}^{\prime}=\alpha_{m+1} /\left(1-X_{m}\right), \alpha_{2}^{\prime}=\alpha_{m+2} /\left(1-X_{m}\right) \cdots$, and $\alpha_{k^{\prime}}^{\prime}=$ $\alpha_{k} /\left(1-X_{m}\right)$, respectively. If the average number of LAs to be paged is $E\left(k^{\prime}\right), C_{p_{2}}$ can be written as

$$
C_{p_{2}}=E\left(k^{\prime}\right) C_{p} .
$$

In a real situation, it may be difficult to obtain the conditional probabilities. However, in the MPBS scheme, it is not necessary for the network to compute them because the network only needs to page the subsequent RAs in the descending order of $\alpha_{m+1}, \alpha_{m+2}, \ldots, \alpha_{k}$. The division by the same positive value does not affect their order. The cost $C_{p_{3}}$ is just the same as in the PBS scheme, and the cost $C_{p_{4}}$ is the same as in the IS-41/GSM MAP scheme. Therefore, we have

$$
C_{p_{3}}=E(k) C_{p}
$$

and

$$
C_{p_{4}}=C_{p}
$$

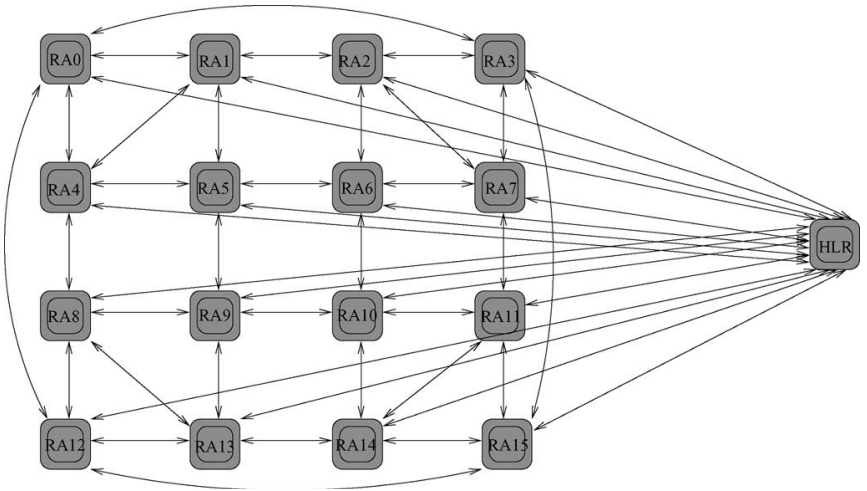

Fig. 3. Network architecture for our simulation study.

Therefore, we can rewrite (5) as

$$
\begin{aligned}
C_{\mathrm{MPBS}}=\frac{\omega_{2} C_{u}}{\rho}+\left[\pi_{1}\left(1.5-Q\left(\frac{T_{\text {threshold }}}{\sigma}\right)\right)\right. \\
\left.+\pi_{2} E\left(k^{\prime}\right)+\pi_{3} E(k)+\pi_{4}\right] C_{p} .
\end{aligned}
$$

In our simulations, we treat the state update messages the same as the registration message and normalize the update cost $C_{u}=1$. The paging cost is usually less than the update cost [11], so we assume the paging cost for one RA is $0<$ $C_{p} \leq C_{u}$. We want to know how the CMR, the paging cost $C_{p}$, the user distribution probability $\alpha_{i}$, and the user out-ofpattern probability $\varphi$ can affect the performance of the PBS and MPBS schemes. In this paper, we assume that there are three representative profile distributions [11]: uniform, linear, and exponential. Denote the conditional probability of a user being in the $i$ th RA in the list as $\beta_{i}=\alpha_{i} /(1-\kappa)$ or $\beta_{i}=\alpha_{i} /(1-\varphi)$. The definitions for the three kinds of distribution are given as follows:

1) uniform distribution: $\beta_{1}=\beta_{2}=\cdots=\beta_{k}=1 / k$;

2) linear distribution: $\beta_{i}=2(k+1-i) / k(k+1)$ for $i \in$ $\{1,2, \ldots, k\} ;$ and

3) exponential distribution: $\beta_{i}=\left[e^{-b i}\left(1-e^{-b}\right)\right] /\left[e^{-b}-\right.$ $\left.e^{-b(k+1)}\right]$ for $i \in\{i, 2, \ldots, k\}$, where $b$ is a constant.

The simulated network architecture is shown in Fig. 3. The network consists of 16 RAs. Each RA has four neighboring RAs. The links between RAs are virtual links, which imply that the user can roam bidirectionally between the connected RAs. The links between RAs and HLR are signaling links. When the user enters an RA, depending on the currently adopted scheme, an update message may be generated and sent to the HLR. The user resides in an RA for some exponentially distributed time with predefined mean and then moves into the next RA. The calls for that user generated in the HLR forms a Poisson process. For simplicity and clarity, we implement the VMN functions into the HLR node. The HLR node will record all the update and paging costs for different schemes at the end of the simulations. In the simulations, we assume that the update cost for every RA is the same. The simulations are event driven. During the initiation, a user is generated randomly in one RA and is set to state 1 . State 4 is different from the other 


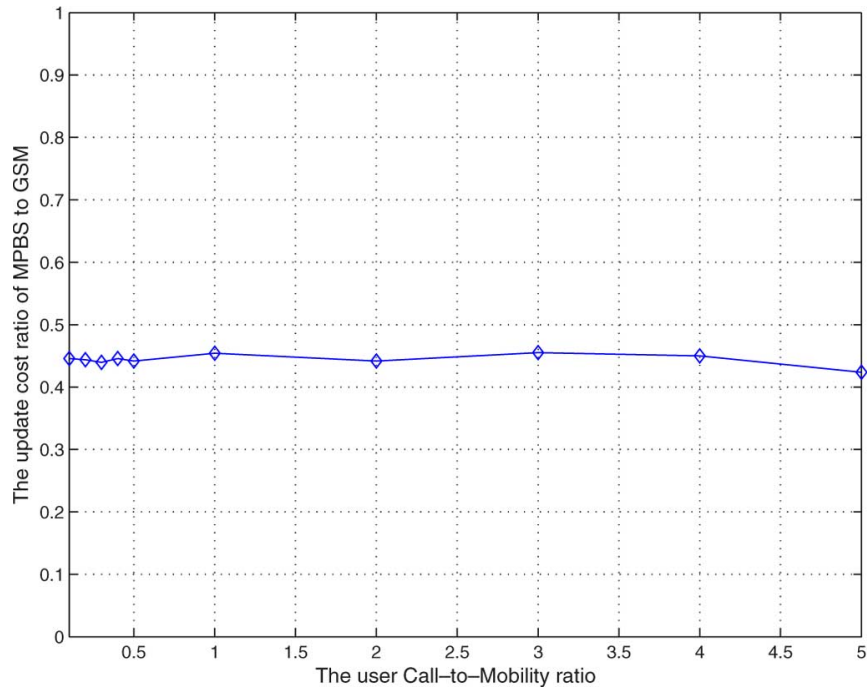

Fig. 4. Location update cost ratio of the MPBS scheme to IS-41/GSM.

states. In state 4 , the user, in fact, does not follow any pattern. Therefore, when the user is in states 1,2 , or 3 , we can say that the user is in-the-pattern, and the user is out-of-pattern when he/she is in state 4 . We also assume that, $90 \%$ of the time, the user is in-the-pattern. The conditional probability of the user in states 1,2 , and 3 are $0.8,0.15$, and 0.05 , respectively. All the simulations collect the user tracking information for $24 \mathrm{~h}$. In order to study the effect of the out-of-pattern probability on performance, the $\varphi$ will change during the simulations. Without loss of generality, we assume that $\alpha_{i}$ and that $t_{i}$ are in the same order in the profile, i.e., $A_{i} \neq A_{j}$ for $i \neq j$.

\section{NUMERICAL RESUlTS AND COMPARISON}

As we have mentioned before, the MPBS can reduce the location update signaling traffic load. Fig. 4 shows the relative location update cost of the MPBS to IS-41/GSM MAP scheme. In this simulation, the out-of-pattern probability is $\varphi=0.1$, and the probabilities of user in the three different states are set as above. In Fig. 4, the update cost for the MPBS scheme is less than half of that for IS-41/GSM MAP, and the CMR does not affect the performance dramatically. The location update cost is usually larger than the paging cost, which is why PBS and MPBS can achieve total saving by reducing the update cost at the expense of increasing paging cost. The advantage of the MPBS scheme is that it can reduce the total cost without increasing the paging cost too much. In both the PBS and MPBS schemes, the network usually pages more than one RAs, trying to find out the user's exact location. In other words, the MPBS and PBS will introduce some delay during the call delivery procedures. In Fig. 5, we can see that the paging delay generated by MPBS is much less than that of PBS. The reason is that, in the PBS scheme, all the RAs in the list could be paged. Usually, the RAs are paged sequentially according to the user distribution probability. Therefore, the delay is the time that elapsed from the instant that the paging message is sent to the first RA to the instant the user responds. The delay can be different with different probability distributions. In Fig. 5, we assume the user profile distribution is uniform. In the MPBS

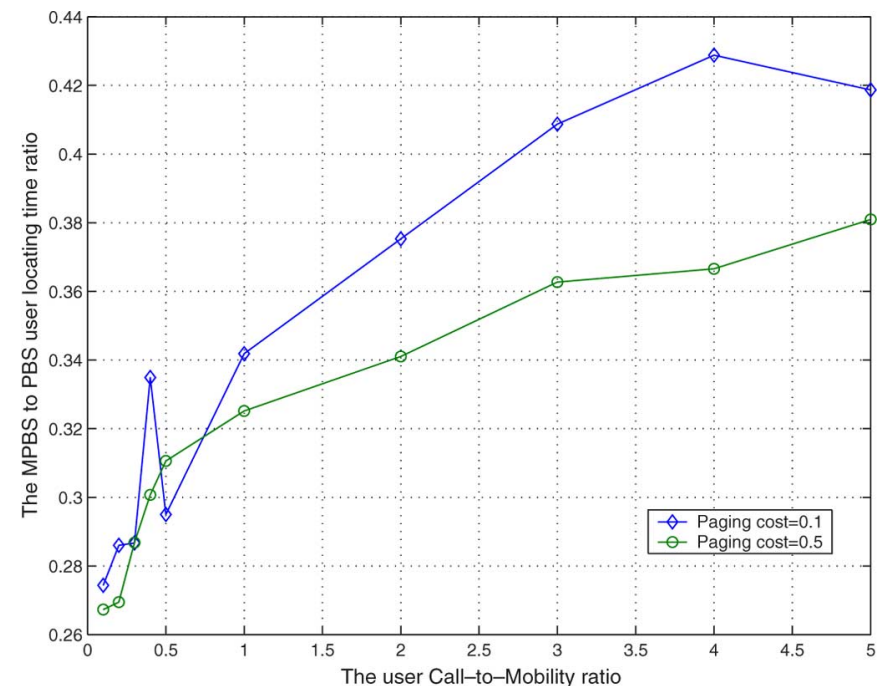

Fig. 5. Comparison of the locating time for MPBS and PBS.

scheme, when the user is in-the-pattern, only when the user is in state 3 do all RAs need to be sequentially paged. When the user is in state 1, only one RA is paged most of the time; when in state 2, only part of $\boldsymbol{A}$ needs to be paged. The paging cost could be considered as the paging delay for one RA. In our simulations, we assume that the paging cost for every RA is equal. As we can see, when the CMR is low, the paging delay for the MPBS scheme is 70\% less than that for the PBS scheme. The reason is that when the CMR is low, the user has a relatively high movement probability; the PBS scheme will page more RAs to find the user, but the MPBS scheme is not affected by this factor. When the CMR is large, the user will stay in an RA for a relative long time; the PBS scheme can find the user with less paging. However, the MPBS total paging cost is still $60 \%$ less than that of the PBS scheme.

Although the MPBS has less paging delay than PBS does, we need to examine the total costs for the two schemes and try to see whether they can achieve better performance than the conventional IS-41/GSM MAP scheme or not.

In Figs. 6-8, we plot the ratios of the MPBS and PBS total costs to the IS-41/GSM MAP total cost, with three different probability distributions and different paging costs. We can see from these figures that the costs for the PBS scheme increase very quickly with the increase of the paging cost. When the paging cost is 0.5 , the PBS scheme can have a saving only when the CMR is very low. In that situation, the user will make a lot of updates to the network, and the update cost is dominant. With the increase of CMR, the paging cost for the PBS scheme plays a more important role; then, the total cost increases fast. The MPBS total cost increases much more slowly with the paging cost. In the wireless multimedia networks, the data service will consume a lot of bandwidth, which makes the paging cost higher. The MPBS total cost increases much slower than PBS with the increase of the paging cost. Another advantage of MPBS is that the cost curves are flatter than PBS in a wide range of CMR when the paging cost is large, which means the MPBS scheme is applicable to different classes of users with a different mobility pattern. In Fig. 8, the total cost of the PBS scheme is a little less than the MPBS scheme when the paging cost is 


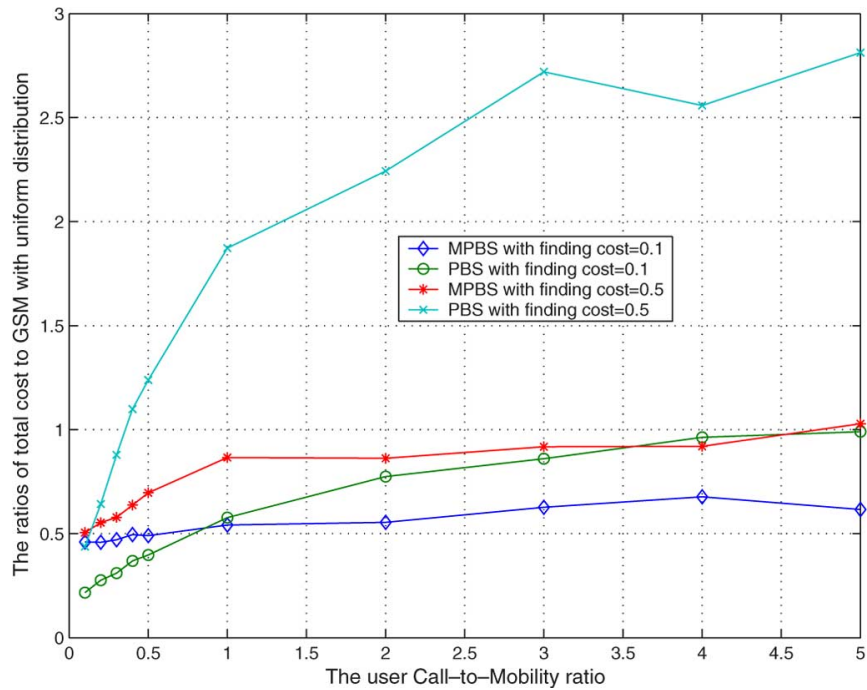

Fig. 6. Total costs of MPBS and PBS to IS-41/GSM with uniform distribution.

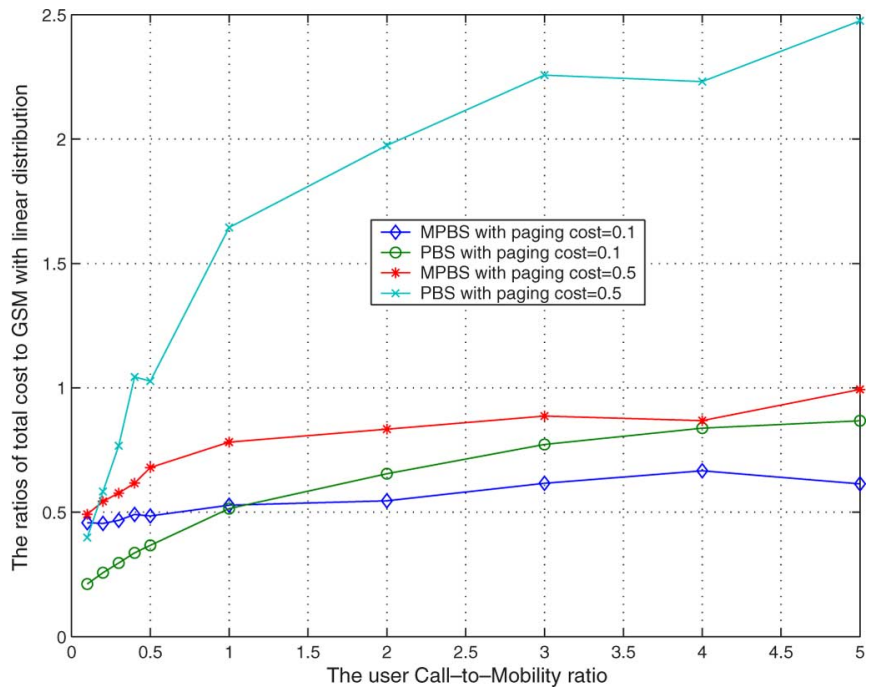

Fig. 7. Total costs of MPBS and PBS to IS-41/GSM with linear distribution.

small. The reason is that, for exponential distribution, the paged RAs are less than other distributions. When the paging cost is small, the total cost may be small, but even with exponential distribution, the PBS total cost increases much faster than the MPBS scheme, with the paging cost.

In the above situations, we assume that, $90 \%$ of the time, the user is in-the-pattern. It is intuitive that the total costs of both the MPBS and PBS schemes are correlated with the outof-pattern probability like $\kappa$ or $\varphi$. Figs. 9 and 10 show how the user's out-of-pattern probability affects the MPBS and PBS performances with different probability distributions. In both figures, we assume $\mathrm{CMR}=1$. The paging cost is 0.1 in Fig. 9 and 0.5 in Fig. 10. In Fig. 9, the total costs for both PBS and MPBS increase with the user out-of-pattern probability. The cost of MPBS is less than PBS. When the paging cost is large, as in Fig. 10, the total costs for PBS are larger than one for all the three distributions. This also proves the conclusion we made before; the PBS scheme can improve the network performance only in very limited conditions. In Fig. 10, the

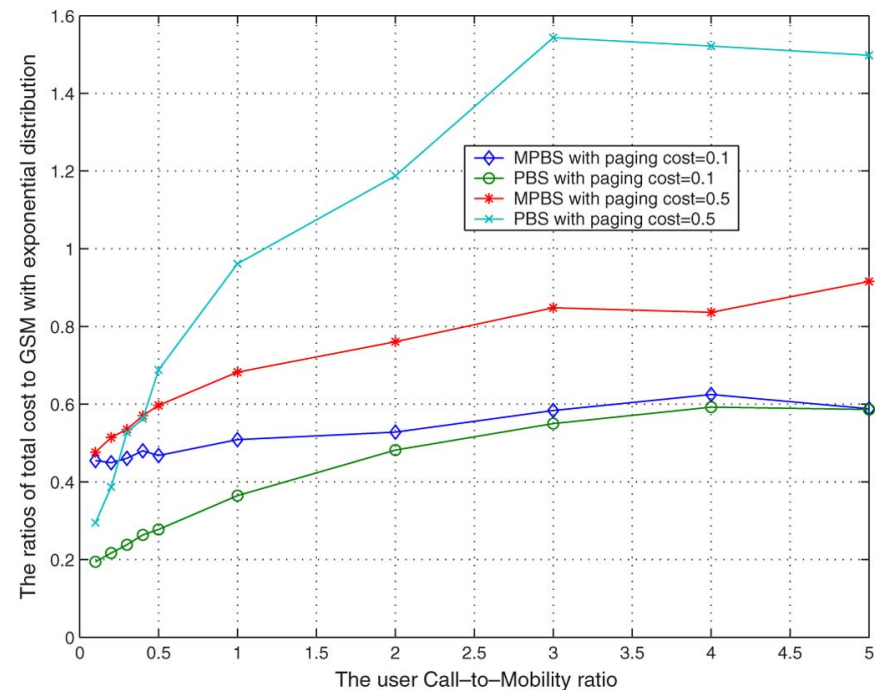

Fig. 8. Total costs of MPBS and PBS to IS-41/GSM with exponential distribution.

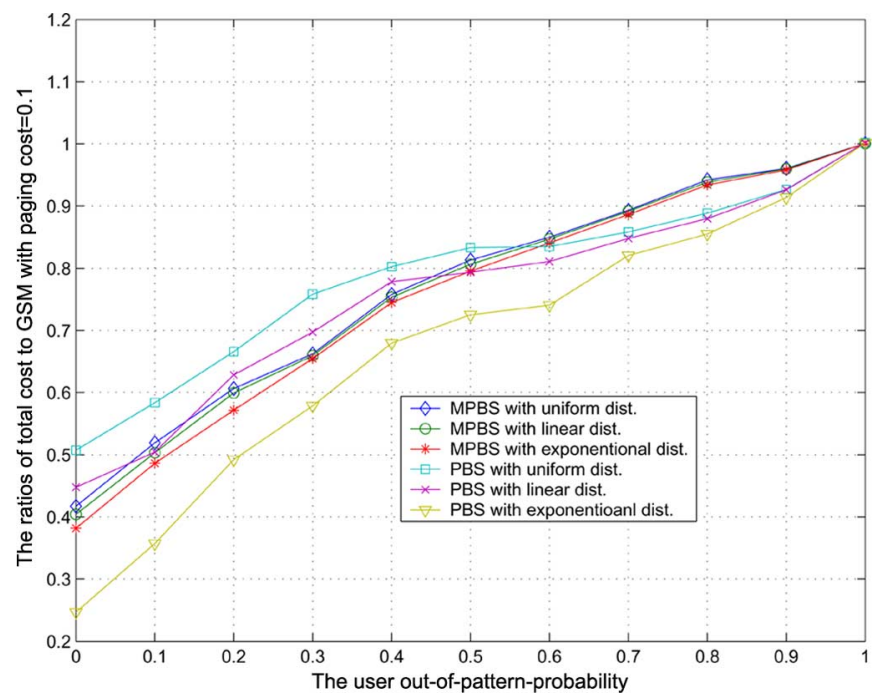

Fig. 9. Effects of user out-of-pattern probability on MPBS and PBS, with paging cost $=0.1$ and $\mathrm{CMR}=1$.

total costs of MPBS are still less than one even if the user has a large probability of getting out of the pattern. In the two figures, all the cost ratios become one when the user out-of-pattern probability is one. The reason is obvious. When the user out-ofpattern is one, the user will not enter any RA in the profile list and needs to register to the network every time he/she moves. The network mobility management scheme is indeed the same as the IS-41/GSM MAP scheme.

\section{CONCLUSION}

For next-generation wireless communication systems, the radio spectrum is scarce. In order to exploit the radio resource efficiently and provide users with high QoS, the LAs become smaller and smaller. The consequence is that the user location update message will consume a lot of bandwidth. This situation becomes worse when the number of users increases. Much research works have been carried out to optimize the network 


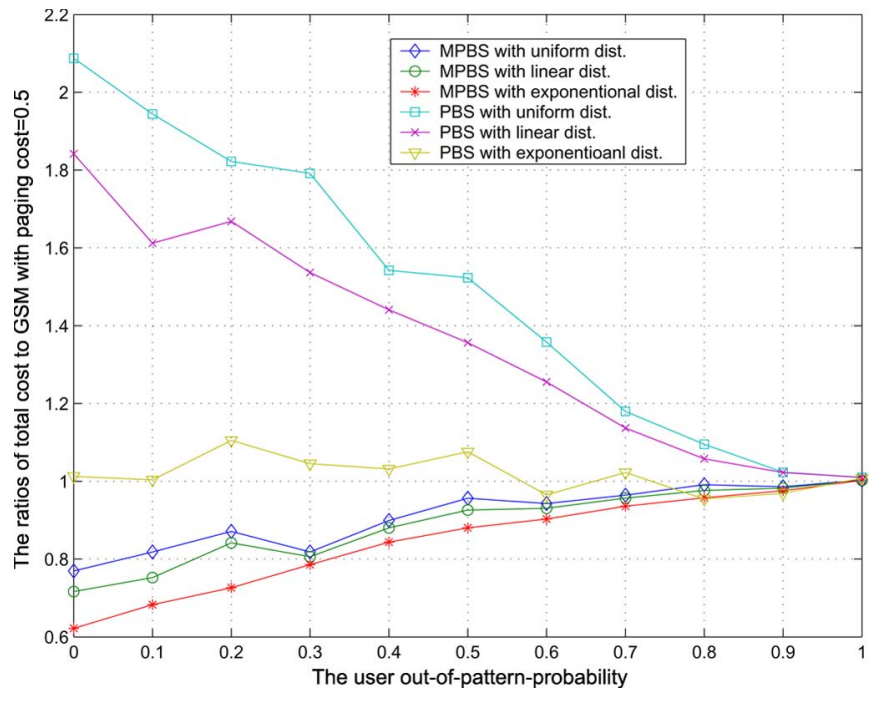

Fig. 10. Effects of user out-of-pattern probability on MPBS and PBS, with paging cost $=0.5$ and $\mathrm{CMR}=1$.

performance in mobility management. In the PBS, no update message is required if the user is in the profile list. Only when the user gets out of the profile are the registration messages sent to the network. When a call arrives for the user, the RAs in the profile are paged according to the descending order of distribution probability until the user is found. In this scheme, the cost for the user location update message can be saved at the expense of the increase of paging. In this paper, we studied the performance of the PBS scheme based on different probability distributions, paging costs, and CMRs. The effect of the user's out-of-pattern probability is also studied. The results show that the PBS scheme works well only for very small CMR, and the total cost increases quickly with the paging cost. We then proposed a new location strategy that is termed as MPBS in this paper. In this scheme, the user mobility time pattern is recorded in the user's profile in addition to the location profile. Simulation results show that, although the MPBS scheme generates more update messages than PBS, the total cost of MPBS is usually significantly less than PBS, and the MPBS scheme is not very sensitive to the increase of the paging cost. One of the most important QoS factors in wireless communication system is the paging delay. Our simulation results show that the paging delay of the MPBS scheme is about 55\% less than the PBS scheme. The results also demonstrate that the MPBS scheme can work well for users with different CMRs.

\section{REFERENCES}

[1] S. Tabbane, "An alternative strategy for location tracking," IEEE J. Sel. Areas Commun., vol. 13, no. 5, pp. 880-892, Jun. 1995.

[2] EIA/TIA, "Cellular radio telecommunications intersystem operations," Tech. Rep. IS-41 (Revision B), 1991.

[3] ETSI, Digital Cellular Telecommunications System (Phase 2+): Mobile Application Part (MAP) Specification (GSM 09.02 version 7.51 Release), 1998.

[4] V. W.-S. Wong and V. C. M. Leung, "Location management for nextgeneration personal communications networks," IEEE Netw., vol. 14, no. 5, pp. 18-24, Sep./Oct. 2000.

[5] I. F. Akyildiz, J. McNair, J. S. M. Ho, H. Uzunalioglu, and W. Wang, "Mobility management in next-generation wireless systems," Proc. IEEE, vol. 87, no. 8, pp. 1347-1384, Aug. 1999.
[6] S. Tabbane, "Location management methods for third-generation mobile system," IEEE Commun. Mag., vol. 35, no. 8, pp. 72-78, Aug. 1997.

[7] I. F. Akyildiz, W. Wang, and Y. Lee, "Location management in 3G/4G wireless systems," in Proc. SPIE-Convergence Inf. ITCom Conf., Denver, CO, Aug. 2001, pp. 1-13.

[8] A. Aljadhai and T. F. Znati, "Predictive mobility support for QoS provisioning in mobile wireless environments," IEEE J. Sel. Areas Commun. vol. 19, no. 10, pp. 1915-1930, Oct. 2001.

[9] S. Choi and K. G. Shin, "Exploiting path/location information for connection admission control in cellular networks," in Proc. IEEE WCNC, Chicago, IL, Sep. 2000, pp. 935-940.

[10] T. Liu, P. Bahl, and I. Chlamtac, "Mobility modeling, location tracking, and trajectory prediction in wireless ATM networks," IEEE J. Sel. Areas Commun., vol. 16, no. 6, pp. 922-936, Aug. 1998.

[11] G. P. Pollini and I. Chih-Lin, "A profile-based location strategy and its performance," IEEE J. Sel. Areas Commun., vol. 15, no. 8, pp. 1415-1424, Oct. 1997.

[12] A. Bhattacharya and S. K. Das, "Le-Zi-update: An information-theoretic framework for personal mobility tracking in PCS networks," Wirel. Netw., vol. 8, no. 2/3, pp. 121-135, Mar./May. 2002.

[13] E. Cayirci and I. F. Akyildiz, "User mobility pattern scheme for location update and paging in wireless systems," IEEE Trans. Mobile Comput., vol. 1, no. 3, pp. 236-246, Jul.-Sep. 2002.

[14] Y. B. Lin and I. Chlamtac, Wireless and Mobile Network Architectures. Hoboken, NJ: Wiley, 2001

[15] W. Ma and Y. Fang, "A new location strategy based on user mobility pattern for PCS," in Proc. IEEE LCN, Tampa, FL, Nov. 2002, pp. 451-457.

[16] — , "Two-level pointer forwarding strategy for location management in PCS networks," IEEE Trans. Mobile Comput., vol. 1, no. 1, pp. 32-45, Jan.-Mar. 2002.

[17] Y. Fang, I. Chlamtac, and Y. B. Lin, "Portable movement modeling for PCS networks," IEEE Trans. Veh. Technol., vol. 49, no. 4, pp. 1356-1363, Jul. 2000 .

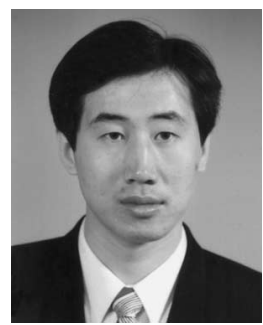

Wenchao Ma (S'99-M'03) received the B.S. and M.S. degrees from Beijing University of Posts and Telecommunications, Beijing, China, and the Ph.D. degree from University of Florida, Gainesville, in 1995,1998 , and 2003, respectively.

From 1998 to 1999 , he was an Engineer with China Telecom. From 1999 to 2000, he was with the Electrical and Computer Engineering Department, New Jersey Institute of Technology, Newark. He also worked with Microsoft Research Asia before 2005 as an Associate Researcher and currently works with the Lenovo Corporate Research and Development, Beijing, as a Research Staffer. His research interests include wireless multimedia networks, mobility management, mobile computing, mobile IP, P2P technology, and broadband wireless access. 


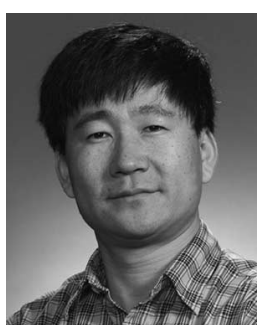

Yuguang Fang received the Ph.D. degree in systems engineering from Case Western Reserve University, Cleveland, OH, in January 1994 and the Ph.D. degree in electrical engineering from Boston University, Boston, MA, in May 1997.

He was an Assistant Professor with the Department of Electrical and Computer Engineering, New Jersey Institute of Technology, Newark, from July 1998 to May 2000. In May 2000, he then joined the Department of Electrical and Computer Engineering, University of Florida, Gainesville, as an Assistant Professor. He got an early promotion to an Associate Professor with tenure in August 2003 and a Professor in August 2005. He will hold the University of Florida Research Foundation (UFRF) Professorship from 2006 to 2009. He has published over 200 papers in refereed professional journals and conferences.

Dr. Fang received the National Science Foundation Faculty Early Career Award in 2001 and the Office of Naval Research Young Investigator Award in 2002. He has served on many editorial boards of technical journals including IEEE TRANSACTIONS ON COMMUNICATIONS, IEEE TRANSACTIONS on Wireless Communications, IEEE TRANSACtions on Mobile Computing, and ACM Wireless Networks.

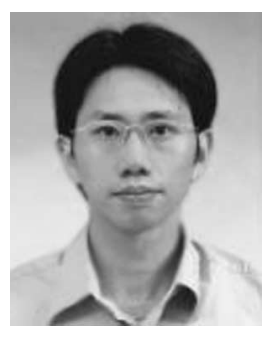

Phone Lin (M'02-SM'06) received the BSCSIE and Ph.D. degrees from National Chiao Tung University, Hsinchu, Taiwan, R.O.C., in 1996 and 2001, respectively.

From August 2001 to July 2004, he was an Assistant Professor with the Department of Computer Science and Information Engineering (CSIE), National Taiwan University, Taipei, Taiwan. Since August 2004, he has been an Associate Professor with the Department of CSIE, National Taiwan University. His current research interests include personal communications services, wireless Internet, and performance modeling.

Dr. Lin is a Guest Editor for the IEEE TRANSACTIONS ON WIRELESS COMMUNiCATIONS special issue on Mobility and Resource Management and a Guest Editor for the ACM/Springer MONET special issue on Wireless Broad Access. He is also an Associate Editorial Member for the WCMC Journal. 